\title{
Pemetrexed-based chemotherapy for non-small-cell lung cancer patients with EGFR exon 20 insertion mutation: a multicenter study
}

\author{
Chun-Wei Xu" ${ }^{1 \#}$, Wen-Xian Wang ${ }^{2 \#}$, Dong Wang ${ }^{1 \#}$, Qi-Ming Wang ${ }^{3}$, Xing-Xiang Pu ${ }^{4}$, You-Cai Zhu ${ }^{5}$, \\ Jian-Hui Huang ${ }^{6}$, Zong-Yang Yu ${ }^{7}$, Zhao-Lei Cui ${ }^{8}$, Xiao-Hui Chen ${ }^{9}$, Jin-Luan Li ${ }^{10}$, Yong Fang ${ }^{11}$, \\ Hong Wang ${ }^{12}$, Wu Zhuang ${ }^{13}$, Shi-Jie Lan ${ }^{14}$, Xin Cai ${ }^{15}$, Yin-Bin Zhang ${ }^{16}$, Wen-Bin Gao ${ }^{17}$, Li-Ping Wang ${ }^{18}$, \\ Ke-Lin She ${ }^{19}$, Chuang-Zhou Rao ${ }^{20}$, Yue-Fen Zhou ${ }^{6}$, Mei-Yu Fang ${ }^{2}$, Li-Yun Miao ${ }^{21}$, Lei Lei ${ }^{2}$, \\ Tang-Feng Lv ${ }^{1}$, Yong Song ${ }^{1}$; on behalf of AME Lung Cancer Collaborative Group
}

${ }^{1}$ Department of Respiratory Medicine, Affiliated Jinling Hospital, Medical School of Nanjing University, Nanjing, China; ${ }^{2}$ Department of Medical Oncology, Chinese Academy of Sciences University Cancer Hospital (Zhejiang Cancer Hospital), Hangzhou China; ${ }^{3}$ Department of Internal Medicine, the Affiliated Cancer Hospital of Zhengzhou University, Henan Cancer Hospital, Zhengzhou, China; ${ }^{4}$ Department of Medical Oncology, Hunan Cancer Hospital, the Affiliated Cancer Hospital of Xiangya School of Medicine, Central South University, Changsha, China; ${ }^{5}$ Department of Thoracic Disease Center, Zhejiang Rongjun Hospital, Jiaxing, China; ${ }^{6}$ Department of Oncology, Lishui Municipal Central Hospital, Lishui, China; ${ }^{7}$ Department of Medical Oncology, the 900th Hospital of the Joint Logistics Team (the Former Fuzhou General Hospital), Fujian Medical University, Fuzhou, China; ${ }^{8}$ Department of Clinical Laboratory, Fujian Cancer Hospital \& Fujian Medical University Cancer Hospital, Fuzhou, China; ${ }^{9}$ Department of Thoracic Surgery, Fujian Cancer Hospital \& Fujian Medical University Cancer Hospital, Fuzhou, China; ${ }^{10}$ Department of Radiotherapy, Xiamen Cancer Hospital, the First Affiliated Hospital of Xiamen University, Xiamen, China; ${ }^{11}$ Department of Oncology, Sir Run Run Shaw Hospital, Hangzhou, China; ${ }^{12}$ Department of Lung Cancer, the Fifth Medical Center, General of PLA, Beijing, China; ${ }^{13}$ Department of Medical Oncology, Fujian Cancer Hospital, Fujian Medical University Cancer Hospital, Fuzhou, China; ${ }^{14}$ Department of Oncology, the First Hospital of Jilin University, Changchun, China; ${ }^{15}$ Department of Oncology, the First Affiliated Hospital of Dalian Medical University, Dalian, China; ${ }^{16}$ Department of Oncology, the Second Affiliated Hospital of Medical College, Xi'an Jiaotong University, Xi'an, China; ${ }^{17}$ Department of Oncology, Shenzhen Luohu People's Hospital, Shenzhen Guangdong, China; ${ }^{18}$ Department of Oncology, Baotou Cancer Hospital, Baotou, China; ${ }^{19}$ Department of Thoracic Surgery, the Central Hospital of Shaoyang City, Shaoyang, China; ${ }^{20}$ Department of Radiation and Chemotherapy, Hwamei Hospital, University of Chinese Academy of Sciences, Ningbo, China; ${ }^{21}$ Department of Respiratory Medicine, Affiliated Drum Tower Hospital, Medical School of Nanjing University, Nanjing, China

Contributions: (I) Conception and design: CW Xu, WX Wang, D Wang; (II) Administrative support: LY Miao, L Lei, Y Song; (III) Provision of study materials or patients: QM Wang, XX Pu, YC Zhu, JH Huang, ZY Yu, ZL Cui, XH Chen, JL Li, Y Fang, H Wang, W Zhuang, SJ Lan, X Cai, YB Zhang, WB Gao, LP Wang, KL She, CZ Rao, YF Zhou, MY Fang; (IV) Collection and assembly of data: CW Xu, WX Wang, D Wang; (V) Data analysis and interpretation: LY Miao, L Lei, Y Song; (VI) Manuscript writing: All authors; (VII) Final approval of manuscript: All authors.

\#These authors contributed equally to this work.

Correspondence to: Yong Song, MD, PhD. Department of Respiratory Medicine, Affiliated Jinling Hospital, Medical School of Nanjing University, 305 Zhongshan Road, Nanjing, China. Email: yong_song6310@yahoo.com; Lei Lei, MD. Department of Chemotherapy, Chinese Academy of Sciences University Cancer Hospital (Zhejiang Cancer Hospital), No. 1 Banshan East Street, Gongshu District, Hangzhou, China. Email: leilei1241@gmail. com; Li-Yun Miao, MD, PhD. Department of Respiratory Medicine, Drum Tower Hospital, Medical School of Nanjing University, 321 Zhongshan Road, Nanjing, China. Email: liyunmiao462@163.com.

Background: Chemotherapy is the major choice for advanced non-small-cell lung cancer (NSCLC) patients with epidermal growth factor receptor exon 20 insertion (EGFR ex20ins). The efficacy of pemetrexed-based with other chemotherapy regimens and EGFR ex20ins subtypes in this population has not been well studied.

Methods: We screened patients with EGFR ex20ins by next-generation sequencing (NGS) from a large cohort. The clinicopathologic and medical information were collected in advanced NSCLC patients with EGFR ex20ins. We also compared the clinical outcomes among patients with different subtypes of EGFR ex20ins.

Results: We retrospectively collected 119 stage IIIB/IV NSCLC patients with EGFR ex20ins from 9142 NSCLC patients across China from June 2013 to December 2018. The subtypes of EGFR ex20ins included 
A767_V769dupASV (33/119, 27.73\%), S768_D770dupSVD (19/119, 15.97\%), N771_H773dupNPH (11/119, 9.24\%), A763_Y764insFQEA (2/119, 1.68\%) and others (54/119, 45.38\%). A total of $64.7 \%$ (77/119) of patients received pemetrexed-based first-line chemotherapy and $13.45 \%(16 / 119)$ of patients received pemetrexed-based second-line chemotherapy. Pemetrexed-based chemo-treated patients had longer median progression-free survival (PFS) than patients without pemetrexed-based chemo-treated (5.5 vs. 3.0 months, $\mathrm{P}=0.0026$ ). Survival data was available for 66 patients and the median overall survival (OS) was 24.7 months. Pemetrexed-based chemo-treated patients had longer OS tendency than patients without pemetrexed-based chemo-treated (25.0 vs. 19.6 months, $\mathrm{P}=0.0769)$. Patients harboring A767_V769dupASV had better OS than other subtypes of EGFR ex20ins but without statistical significance $(\mathrm{P}=0.0676)$. Multivariate analysis revealed that histological type of NSCLC and bone-metastasis before treatment were independent prognostic factors for OS in all patients after adjusting all characteristic and treatment factors $(\mathrm{P}<0.05)$.

Conclusions: To the best of our knowledge, it is the largest cohort study of advanced NSCLC patients with EGFR ex20ins across China. Pemetrexed-based treatment could have better control of disease than non-pemetrexed-based chemotherapies in this population. Furthermore, more effective agents are expected for patients harboring EGFR ex20ins.

Keywords: Exon 20 insertion (ex20ins); non-small-cell lung cancer (NSCLC); chemotherapy; pemetrexed

Submitted Mar 04, 2020. Accepted for publication Aug 10, 2020.

doi: $10.21037 /$ tlcr-20-382

View this article at: http://dx.doi.org/10.21037/tlcr-20-382

\section{Introduction}

Lung cancer is the leading cause of cancer-related deaths in the worldwide for both male and female (1). The discovery of epidermal growth factor receptor $(E G F R)$ mutation as oncogenic driver and predictor for sensitivity of tyrosine kinase inhibitors (TKIs) rather than chemotherapy has changed the clinical practice in patients with classical-EGFR mutant advanced non-small-cell lung cancer (NSCLC) $(2,3)$. The EGFR exon 20 insertion (ex20ins) mutation is the most common mutation in non-classical EGFR mutations, accounting for about $1.5 \%$ to $2.5 \%$ of all lung cancers, and about $10 \%(1-17 \%)$ of all EGFR mutations $(4,5)$. Most of these mutations are typically located near or after the C-helix of tyrosine kinase domain of EGFR ranging from $\mathrm{M} 766$ residues to $\mathrm{C} 775$, and a small portion in the middle of the C-helix (E762 to Y764) (4,5). Patients with EGFR ex20ins have similar clinical features to those with classical EGFR mutations but a worse prognosis (6). EGFR ex20ins could change the binding conformation between the first and second generations of TKIs and the active TK domain which associated with primary resistance to TKIs $(5,7)$. Therefore, most of patients harboring EGFR ex20ins mutations could barely benefit from EGFR-TKIs therapy except for A763_Y764insFQEA $(5,8,9)$.
Previous studies have shown that patients with EGFR ex20ins could have better response to chemotherapy than TKIs $(6,10)$. However, no standard chemotherapy recommendation been established yet. The platinum-based chemotherapy is the commonly used first-line therapy for this population in clinical practice. Meanwhile, pemetrexedbased chemotherapy could have better efficacy and tolerance than platinum-based chemotherapy in patients with advanced un-targetable NSCLC (11). Given the lack of effective target therapy in most NSCLC patients with EGFR ex20ins mutations, optimization of first-line chemotherapy could be significant in medical care.

In this study, we launched a large retrospective nationwide study of the clinical characteristics and outcomes in advanced NSCLC patients with EGFR ex20ins to explore the performance of pemetrexed-based chemotherapy.

We present the following article in accordance with the STROBE reporting checklist (available at http://dx.doi. org/10.21037/tlcr-20-382).

\section{Methods}

\section{Study populations}

We conducted a multicenter, retrospective study involving 


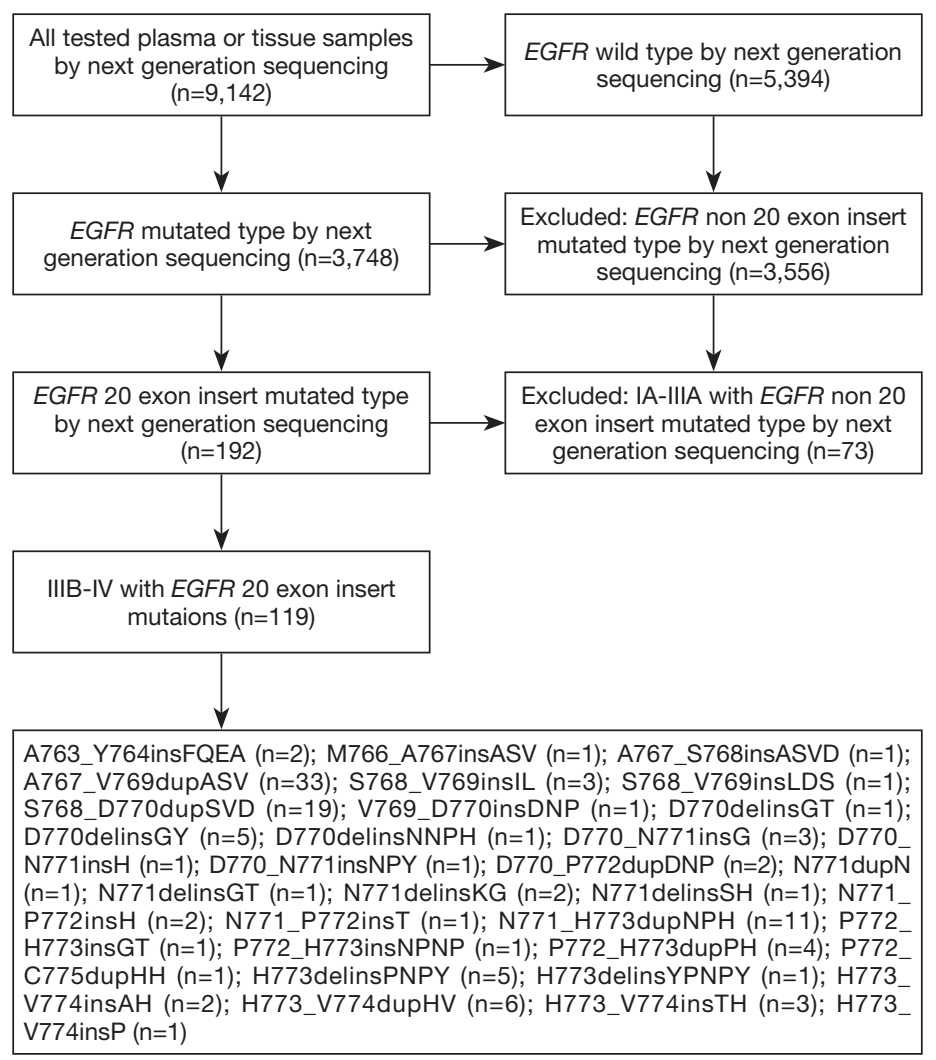

Figure 1 Flow chart of this cohort study. EGFR, epidermal growth factor receptor.

several medical centers in many different areas, including the southern, northern, eastern, and western parts of China between 2013 and 2018. The genetic alterations results obtained from 9,142 plasma or tissue samples of NSCLC patients who underwent the next-generation sequencing (NGS) detection for EGFR mutation screening. A total of 119 advanced NSCLC patients with EGFR ex20ins met the enrollment criteria and were further analyzed in this study (Figure 1). The lung tumor histology was classified according to the World Health Organization criteria (2015 version) (12). Lung cancer clinical staging was performed in all patients according to the 8th TNM classification (13). Response to treatment was assessed according to Response Criteria in Solid Tumors (RECIST) 1.1 (14). All procedures performed in this study were in accordance with the Declaration of Helsinki (as revised in 2013) and the study was approved by the Drum Tower Hospital, Medical School of Nanjing University Ethics Committee (No. 2019004) and written informed consent was taken from all the patients.

\section{Targeted NGS}

DNA from formalin-fixed, paraffin-embedded tumor tissue and matched blood samples was extracted. Comprehensive genomic profiling was performed by NGS with a 381 cancer related gene panel covering the whole exons of EGFR gene at a mean coverage depth of $>800 \times$. Genomic DNA sequencing libraries were prepared using the protocols recommended by the Illumina TruSeq DNA Library Preparation Kit. For samples close to the minimum input requirement, additional pre-capture PCR cycles were performed to generate sufficient PCR product for hybridization. The libraries were hybridized to customdesigned probes (Integrated DNA Technology) including all exons of 170 genes and selected intron of $A L K, R E T$ and ROS1 for the detection of genomic rearrangements. DNA sequencing was performed on a HiSeq3000 sequencing system (Illumina, San Diego, CA, USA) with $2 \times 75$ bp paired-end reads. The reads were aligned to the human genome build GRCh37 using a Burrows-Wheeler aligner (BWA). Somatic single nucleotide variant (sSNV) and indel 
Table 1 Clinical characteristics of the study population $(\mathrm{n}=119)$

\begin{tabular}{|c|c|c|}
\hline Characteristics & Number & Percentage (\%) \\
\hline \multicolumn{3}{|l|}{ Gender } \\
\hline Male & 83 & 69.75 \\
\hline Female & 36 & 30.25 \\
\hline \multicolumn{3}{|l|}{ Age (years) } \\
\hline$\leq 60$ & 78 & 65.55 \\
\hline$>60$ & 41 & 34.45 \\
\hline \multicolumn{3}{|l|}{ Smoking status } \\
\hline No & 59 & 49.58 \\
\hline Yes & 60 & 50.42 \\
\hline \multicolumn{3}{|l|}{ Histology } \\
\hline Adenocarcinoma & 107 & 89.92 \\
\hline Non-adenocarcinoma & 12 & 10.08 \\
\hline \multicolumn{3}{|l|}{ ECOG PS } \\
\hline $0-1$ & 97 & 81.51 \\
\hline$\geq 2$ & 22 & 18.49 \\
\hline \multicolumn{3}{|l|}{ Clinical stage } \\
\hline IIIB & 11 & 9.24 \\
\hline IV & 108 & 90.76 \\
\hline \multicolumn{3}{|c|}{ Brain metastases before treatment } \\
\hline No & 87 & 73.11 \\
\hline Yes & 32 & 26.89 \\
\hline \multicolumn{3}{|c|}{ Bone metastases before treatment } \\
\hline No & 73 & 61.34 \\
\hline Yes & 46 & 38.66 \\
\hline \multicolumn{3}{|c|}{ EGFR exon 20 insert mutant group } \\
\hline A767_V769dupASV & 33 & 27.73 \\
\hline S768_D770dupSVD & 19 & 15.97 \\
\hline N771_H773dupNPH & 11 & 9.24 \\
\hline A763_Y764insFQEA & 2 & 1.68 \\
\hline Others & 54 & 45.38 \\
\hline \multicolumn{3}{|l|}{ EGFR-TKI } \\
\hline No & 108 & 90.76 \\
\hline Yes & 11 & 9.24 \\
\hline \multicolumn{3}{|l|}{ First-line chemotherapy } \\
\hline Pemetrexed-based & 77 & 64.71 \\
\hline
\end{tabular}

Table 1 (continued)
Table 1 (continued)

\begin{tabular}{lcc}
\hline Characteristics & Number & Percentage (\%) \\
\hline Non-pemetrexed & 42 & \\
Second-line chemotherapy & & \\
Pemetrexed-based & 16 & 13.45 \\
Non-pemetrexed based & 103 & 86.55 \\
\hline EGFR, epidermal growth factor receptor; TKI, tyrosine kinase \\
inhibitor.
\end{tabular}

calls were generated using MuTect and GATK, respectively. Somatic copy number alterations were identified with CONTRA. Genomic rearrangements were identified by the software developed in house analyzing chimeric read pairs.

\section{Statistical analysis}

Kaplan-Meier method was adopted to estimate and plot the survival endpoints for progression-free survival (PFS) and overall survival (OS) in this study. The Cox proportional hazards regression model was used to evaluate the impact of selected variables on OS. The differences were accessed by the two-sided $\mathrm{P}$ values with a significance level of 0.05 . All analyses were performed using $\mathrm{R}$ statistics package ( $\mathrm{R}$ version 3.4.0; $\mathrm{R}$ : The R-Project for Statistical Computing, Vienna, Austria).

\section{Results}

\section{Characteristics of patients with EGFR ex20ins}

A total of 119 patients with advanced NSCLC and EGFR ex20ins were enrolled in this study. There were 83 males and 36 females, with a median age of 59 (range, 35-83) years. The number ever and never smokers were 59 and 60 , respectively. Eleven patients were stage IIIB and 108 were stage IV according to the 8th TNM classification (13). Thirty-two patients had brain metastases before treatment and 46 patients had bone metastases before treatment. All patients have ever received chemotherapy and 11 of them have received EGFR-TKIs treatment. Four patients received osimertinib and seven patients received icotinib as second-line or further treatment. The characteristics of patients are listed in Table 1.

\section{Subtypes of EGFR ex20ins}

EGFR ex20ins mutations were identified in $5.1 \%$ 

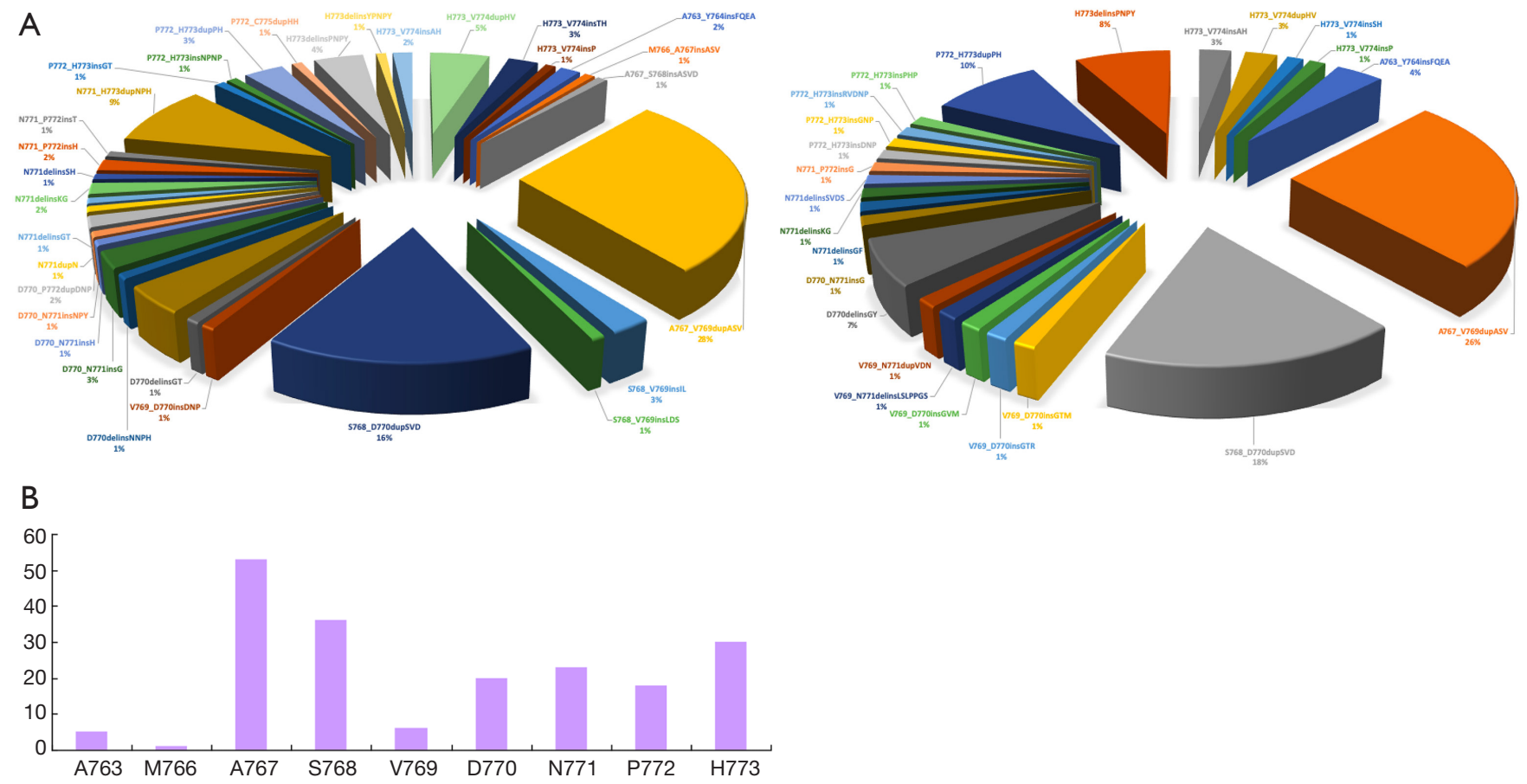

Figure 2 Distribution of EGFR ex20ins mutants in 73 NSCLC patients (I-IIIA) according to different classes by functional classification [(A) left side)] and 119 advanced NSCLC patients (IIIB-IV) according to different classes by functional classification [(A) right side)]. Number of ex20ins mutants (B). EGFR ex20ins, epidermal growth factor receptor exon 20 insertion; NSCLC, non-small-cell lung cancer.

$(192 / 3,748)$ of $E G F R$-mutant NSCLC and $2.1 \%(192 / 9,142)$ of all NSCLC cases. Of 192 patients with EGFR ex20ins in all stages NSCLC, 119 patients with stage IIIB/IV NSCLC were identified and enrolled in this study. The overview of EGFR ex20ins distribution in these two groups of patients were shown in Figure $2 \mathrm{~A}$. The most common insertion sites in EGFR exon 20 in NSCLC was A767 (20/119, $16.81 \%$ ), followed by S768, H773, N771, D770, D772, V769, A763 and M767 (Figure 2B). The frequent subtypes of EGFR ex20ins included A767_V769dupASV (33/119, 27.73\%), S768_D770dupSVD (19/119, 15.97\%), N771_ H773 dupNPH (11/119, 9.24\%), A763_Y764insFQEA $(2 / 119,1.68 \%)$ and others $(54 / 119,45.38 \%)$ (Figure 2$)$. The most common co-mutations were TP53 (61/119, 51.3\%). The co-mutations with NSCLC driver genes included KRAS (6/119, 5.04\%), ERBB2 (2/119, 1.68\%), ALK (2/119, $1.68 \%), B R A F(4 / 119,3.36 \%)$ and $R E T(8 / 119,6.72 \%)$.

\section{Efficacy of chemotherapy}

Seventy-seven patients received a first-line pemetrexedbased chemotherapy, including pemetrexed/platinum $(n=66)$ and pemetrexed monotherapy $(n=11)$. Others received paclitaxel/platinum $(\mathrm{n}=32)$ and gemcitabine/ platinum $(n=10)$. The initial dosages of chemotherapy were pemetrexed $500 \mathrm{mg} / \mathrm{m}^{2}$ day 1 ; gemcitabine $1,000-$ $1,250 \mathrm{mg} / \mathrm{m}^{2}$ day 1,8 ; paclitaxel $175 \mathrm{mg} / \mathrm{m}^{2}$ day 1 ; carboplatin area under the curve (AUC) $=5$ day 1 ; cisplatin $75 \mathrm{mg} / \mathrm{m}^{2}$ day 1 . The objective response rate (ORR) and disease control rate (DCR) of first-line pemetrexedbased chemotherapy were $41.56 \%(32 / 77)$ and $75.32 \%$ (58/77), respectively. The ORR and DCR of the other chemotherapies were $30.95 \%$ (13/42) and 54.76\% (23/42), respectively.

\section{Survival analysis}

In all patients with EGFR ex20ins, first-line pemetrexedbased chemo-treated patients had longer median PFS than patients without pemetrexed-based chemo treated (5.5 vs. 3.0 months, $\mathrm{P}=0.0026$ ) (Figure 3 A). Pemetrexedbased chemo treated patients had longer OS tendency than patients without pemetrexed-based chemo treated (25.0 vs. 19.6 months, $\mathrm{P}=0.0769$ ) (Figure $3 B$ ). The OS follow-up data were available for 66 patients and the median OS was 24.7 months (Figure 4A). No significant differences of 

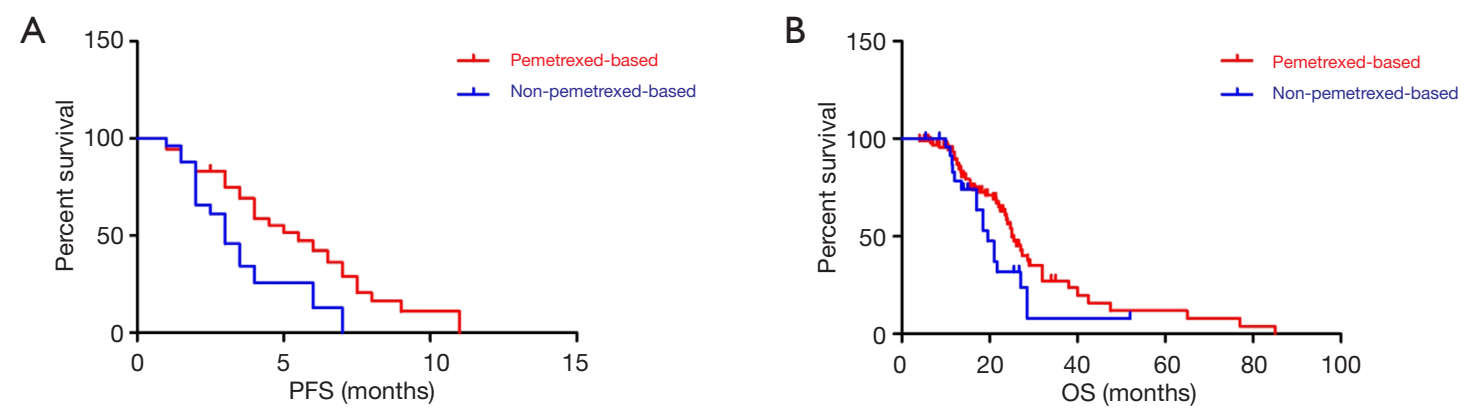

Figure 3 Survival analysis. (A) PFS of all patients with EGFR ex20ins mutants treated with pemetrexed-based or other chemotherapy (5.5 vs. 3.0 months, $\mathrm{P}=0.0026$ ); (B) OS of all patients with EGFR ex20ins mutants ever treated with pemetrexed-based or non-pemetrexed-based chemotherapy (25.0 vs. 19.6 months, $\mathrm{P}=0.0769)$. EGFR ex20ins, epidermal growth factor receptor exon 20 insertion; PFS, progression-free survival; OS, overall survival.
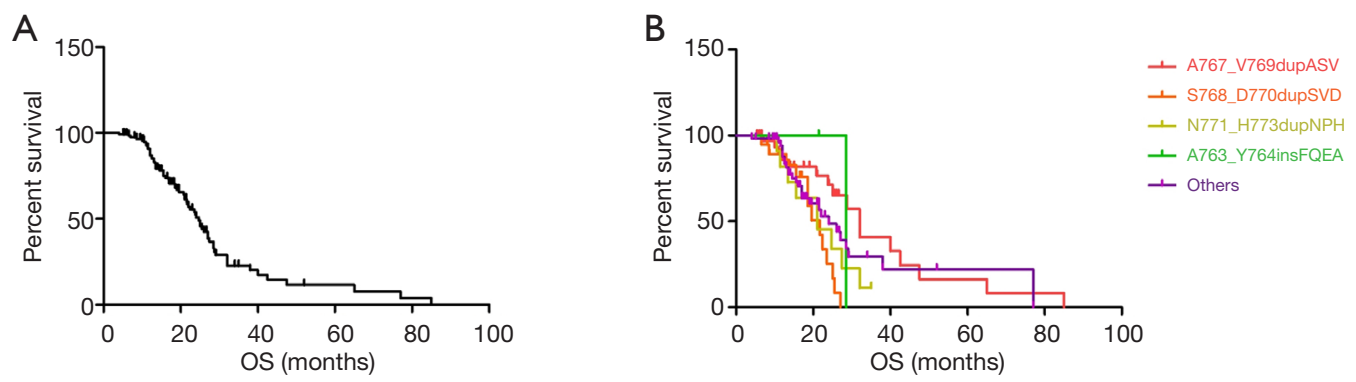

Figure 4 Survival analysis. (A) The median OS of the 119 patients was 24.7 months; (B) a significant improvement of OS was seen in lung cancer patients with A767_V769dupASV, S768_D770dupSVD, N771_H773dupNPH, A763_Y764insFQEA with others (32.0 vs. 21.7, 21.0, 28.5 and 24.0 months, $\mathrm{P}=0.0676$ ). OS, overall survival.

OS were found among patients with different subtypes of EGFR ex20ins ( $\mathrm{P}=0.0676$ ) (Figure 4B). Multivariate analysis revealed that histological type of NSCLC and bonemetastasis before treatment were independent prognostic factors for OS in all patients after adjusting all characteristic and treatment factors $(\mathrm{P}<0.05$; Table 2).

\section{Discussion}

As far as we know, this is the largest Chinese cohort study of advanced NSCLC patients with EGFR ex20ins and analysis of survival benefit from pemetrexed-based chemotherapy. The prevalence of EGFR ex20ins in all EGFR mutants and all NSCLC were $5.1 \%$ and $2.1 \%$, respectively that were concordant with other studies (15-17). The clinical feature of patients with EGFR ex20ins mutations was also discussed in our study. The most common subtype of $E G F R$ ex20ins mutation was A767_V769dup and the co-mutation gene was TP53 that consistent with the conclusion from another study in the Chinese population (15). We found that pemetrexed-based chemotherapy could prolong the PFS of advanced NSCLC patients with EGFR ex20ins compared to non-pemetrexed-based chemotherapy which is consistent with one study from Taiwan (17). The tendency of longer OS was also observed in the pemetrexed-based chemo-treated patients than other chemo-treated patients (25.0 vs. 19.6 months, $\mathrm{P}=0.0769$ ). The patients harboring A767_V769dupASV appeared to have a better OS than other subtypes of EGFR ex20ins $(\mathrm{P}=0.0676)$ although the subtypes of EGFR ex20ins have no independent impact on OS by multivariant analysis ( $\mathrm{HR}=1.1, \mathrm{P}=0.274)$.

Crystallographic studies have shown the EGFR ex20ins mutations could destabilize the inactive conformation of EGFR and induce its constitutive activation $(18,19)$. Up to $12 \%$ of patients with EGFR ex20ins mutations in all EGFRmutant NSCLC patients has been reported in a large case series study, EGFR ex20ins in NSCLC is featured with a wide variety of subtypes and unique molecular alterations 
Table 2 Results of multivariate Cox regression model in EGFR exon 20 insert mutation patients

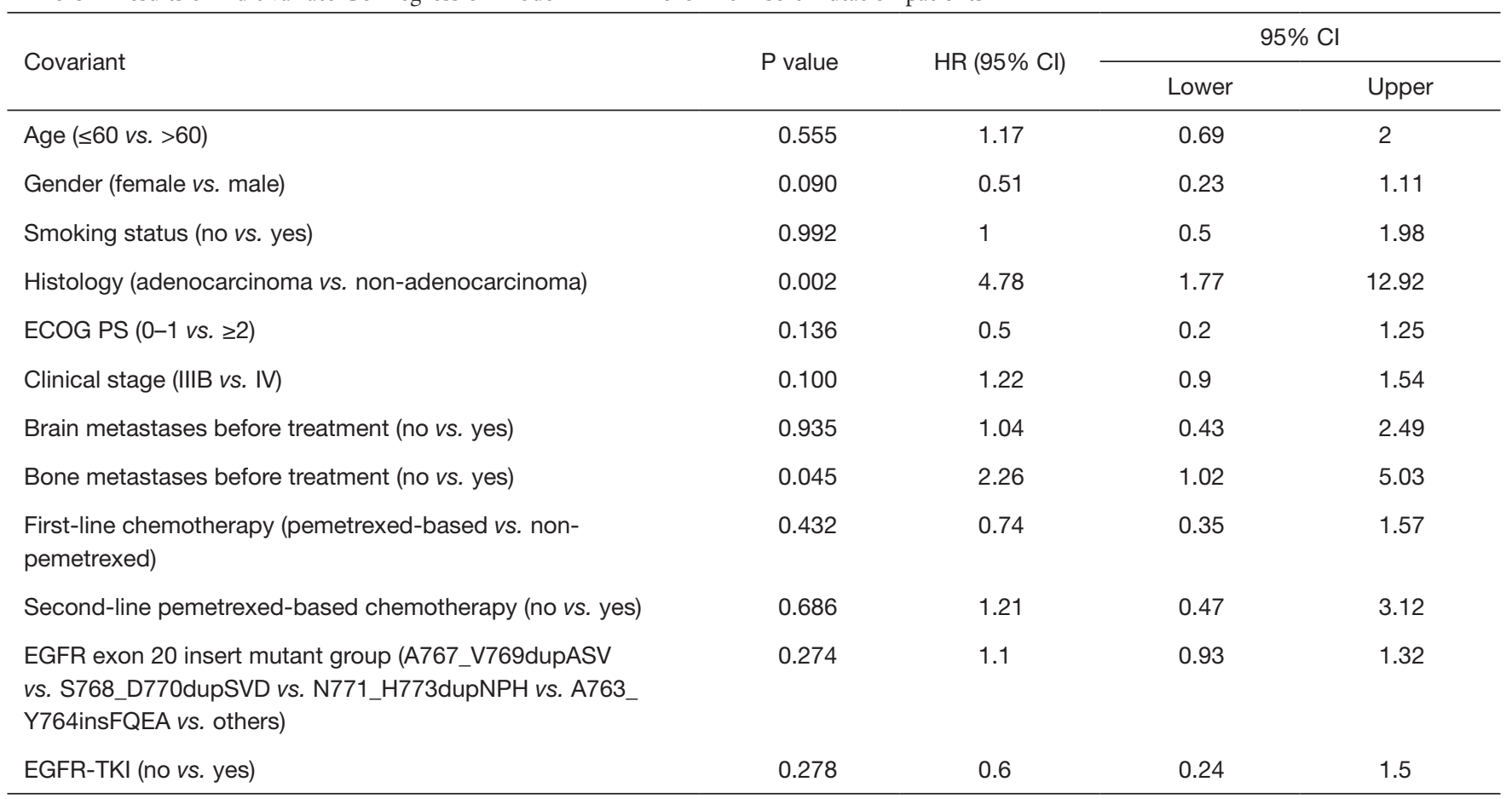

EGFR, epidermal growth factor receptor; TKI, tyrosine kinase inhibitor.

of co-existing genomic alterations (16). The A767_ V769dupASV was the most common subtype of EGFR ex20ins identified in our study which is consistent with the previous study in Chinese cohort (15). Besides, the co-exist frequency of other oncogenic driver mutations in patients harboring EGFR ex20ins mutations was also very low in this study (15). The patients with A767_V769dupASV had the longest OS in all common subtypes of EGFR ex20ins in our study whilst patients with S768_D770dupSVD and N771_ H773dupNPH had the worst OS less than 2 years. Most of the A767_V769dupASV carriers received chemotherapy except one patient also accepted osimertinib after first-line pemetrexed-based chemotherapy. Osimertinib has been demonstrated to be more potent than erlotinib for the A767_V769dupASV mutant in vitro (20).

Chemotherapy remains to be the standard first-line therapy for NSCLC patients with EGFR exon20ins since most of them have a poor response to TKIs (10). Pemetrexed is a multitargeted antifolate and it could be used as monotherapy or combination with platinum in first or subsequent lines or maintenance option in advanced non-squamous NSCLC patients $(21,22)$. In Wu et al.'s study, pemetrexed-based therapies were observed to have better PFS (6.2 vs. 2.7 months; $\mathrm{P}<0.001)$ and $\mathrm{OS}(28.0$ vs. 15.4 months; $\mathrm{P}=0.009)$ than other regimens without pemetrexed (17). Their conclusion was very close to ours. Although A763_Y764insFQEA mutant was one exception that has been identified as indicators for TKIsensitive EGFR exon20ins mutant in literature $(9,10,23)$, little is known about the efficacy of chemotherapy in this subpopulation. Lin et al. (24) reported clinical outcome of six advanced NSCLC patients with A763_Y764insFQEA, one of them received first-line pemetrexed + cisplatin harvested the best response to PR and 28 months of PFS and $>57.0$ months of OS; while another patient received gemcitabine + cisplatin reached SD as best response and the PFS and OS were only 7.0 and 17.0 months, respectively. Both of A763_Y764insFQEA carriers in our study had accepted icotinib during follow-up, and they had PFS less than 6.0 months after first-line chemotherapy but longer than 2 years OS. The association between different subtypes of EGFR exon20ins and efficacy of chemotherapy still needs to be determined in the future, especially in those target therapy-resistant subtypes.

Despite general poor response to targeted therapy in patients with EGFR ex20ins, comparable OS after systemic chemotherapy was reported in all patients with EGFR-mutant lung adenocarcinomas previously (26 vs. 31 months, $\mathrm{P}=0.53$ ) (10). Herein, we aimed not 
only to present the superior efficacy of pemetrexed-based chemotherapy than non-pemetrexed-based chemotherapy in the first-line setting of EGFR exon20ins carriers but also claim a needy of more effective targeted therapies for those who could not benefit from chemotherapy and approved target therapies.

There are two major limitations should be noticed. Firstly, this is a retrospective study and we enrolled patients with late stages of NSCLC only that the bias of patient selection could not be neglected. Giving the low incidence and complex variants of EGFR ex20ins, we presented the largest study of advanced NSCLC patients with EGFR ex20ins from the nation-wide genetic-screening database as far as we know. Secondly, we have not collected all the treatment information after second-line chemotherapy which could influence the outcome of patients with EGFR ex20ins. However, more than two-thirds of patients have received pemetrexed-based chemotherapy and achieved significant survival benefits compared with other chemotherapy which has not been reported in the Chinese population before. Optimizing chemotherapy could be meaningful to those TKIs-resistant population.

\section{Conclusions}

To the best of our knowledge, it is the largest cohort study of advanced NSCLC patients with EGFR ex20ins from China. Pemetrexed-based treatment could have better control of disease than non-pemetrexed-based chemotherapies in this population. Furthermore, more novel and effective agents are expected to ice-break the limitation of survival benefit from toxic chemotherapy.

\section{Acknowledgments}

Funding: This study was supported in part by grants from the Medical Scientific Research Foundation of Zhejiang Province of China (2019RC027), Zhejiang Public Welfare Technology Research Program (LGJ20H160001), Science and Technology Planning Project of Zhejiang Province (LGF19H160002), Zhejiang Traditional Chinese Medicine Science Fund Project (2020ZB037), Clinical Medical Technology Innovation Project of Hunan Province (2017SK51410), Scientific Research Foundation of Zhejiang Medical Association (2019ZYC-A76) and Xisike-Hanson Cancer Research Foundation (Y-HS2019-20).

\section{Footnote}

Reporting Checklist: The authors have completed the STROBE reporting checklist. Available at http://dx.doi. org/10.21037/tlcr-20-382

Data Sharing Statement: Available at http://dx.doi. org/10.21037/tlcr-20-382

Conflicts of Interest: All authors have completed the ICMJE uniform disclosure form (available at http://dx.doi. org/10.21037/tlcr-20-382). The authors have no conflicts of interest to declare.

Ethical Statement: The authors are accountable for all aspects of the work in ensuring that questions related to the accuracy or integrity of any part of the work are appropriately investigated and resolved. All procedures performed in this study were in accordance with the Declaration of Helsinki (as revised in 2013) and the study was approved by the Drum Tower Hospital, Medical School of Nanjing University Ethics Committee (No. 2019004) and written informed consent was taken from all the patients.

Open Access Statement: This is an Open Access article distributed in accordance with the Creative Commons Attribution-NonCommercial-NoDerivs 4.0 International License (CC BY-NC-ND 4.0), which permits the noncommercial replication and distribution of the article with the strict proviso that no changes or edits are made and the original work is properly cited (including links to both the formal publication through the relevant DOI and the license). See: https://creativecommons.org/licenses/by-nc-nd/4.0/.

\section{References}

1. Torre LA, Siegel RL, Jemal A. Lung cancer statistics. Adv Exp Med Biol 2016;893:1-19.

2. Tanaka S, Uchino J. Erlotinib as standard adjuvant therapy for resectable EGFR mutation-positive non-small cell lung cancer. Transl Lung Cancer Res 2019;8:S369-72.

3. Chen D, Chu T, Chang Q, et al. The relationship between preliminary efficacy and prognosis after first-line EGFR tyrosine kinase inhibitor (EGFR-TKI) treatment of advanced non-small cell lung cancer. Ann Transl Med 2019;7:195.

4. Tanaka S, Uchino J. Erlotinib as standard adjuvant therapy 
for resectable EGFR mutation-positive non-small cell lung cancer. Transl Lung Cancer Res 2019;8:S369-72.

5. Tu HY, Ke EE, Yang JJ, et al. A comprehensive review of uncommon EGFR mutations in patients with non-small cell lung cancer. Lung Cancer 2017;114:96-102.

6. Oxnard GR, Lo PC, Nishino M, et al. Natural history and molecular characteristics of lung cancers harboring EGFR exon 20 insertions. J Thorac Oncol 2013;8:179-84.

7. Cheng L, Alexander RE, MacLennan GT, et al. Molecular pathology of lung cancer: key to personalized medicine. Mod Pathol 2012;25:347-69.

8. Wu JY, Wu SG, Yang CH, et al. Lung cancer with epidermal growth factor receptor exon 20 mutations is associated with poor gefitinib treatment response. Clin Cancer Res 2008;14:4877-82.

9. Voon PJ, Tsui DWY, Rosenfeld N, et al. EGFR exon 20 insertion A763-Y764insFQEA and response to erlotinibletter. Mol Cancer Ther 2013;12:2614-5.

10. Naidoo J, Sima CS, Rodriguez K, et al. Epidermal growth factor receptor exon 20 insertions in advanced lung adenocarcinomas: clinical outcomes and response to erlotinib. Cancer 2015;121:3212-20.

11. Tomasini P, Greillier L, Khobta N, et al. The place of pemetrexed in the management of non-small-cell lung cancer patients. Expert Rev Anticancer Ther 2013;13:257-66.

12. Travis WD, Brambilla E, Burke A, et al. WHO classification of tumours of the lung, pleura, thymus and heart. 4th ed. Lyon: International Agency for Research on Cancer, 2015.

13. Detterbeck FC, Chansky K, Groome P, et al. The IASLC lung cancer staging project: methodology and validation used in the development of proposals for revision of the stage classification of NSCLC in the forthcoming (eighth) edition of the TNM classification of lung cancer. J Thorac Oncol 2016;11:1433-46.

14. Eisenhauer EA, Therasse P, Bogaerts J, et al. New response evaluation criteria in solid tumours: revised RECIST guideline (version 1.1). Eur J Cancer 2009;45:228-47.

15. Fang $\mathrm{W}$, Huang $\mathrm{Y}$, Hong $\mathrm{S}$, et al. EGFR exon 20 insertion mutations and response to osimertinib in non-small-cell lung cancer. BMC Cancer 2019;19:595.

16. Riess JW, Gandara DR, Frampton GM, et al. Diverse EGFR Exon 20 insertions and co-occurring molecular alterations identified by comprehensive genomic profiling of NSCLC. J Thorac Oncol 2018;13:1560-8.
17. Wu JY, Yu CJ, Shih JY. Effectiveness of treatments for advanced non-small-cell lung cancer with exon 20 insertion epidermal growth factor receptor mutations. Clin Lung Cancer 2019;20:e620-30.

18. Yasuda H, Park E, Yun CH, et al. Structural, biochemical, and clinical characterization of epidermal growth factor receptor (EGFR) exon 20 insertion mutations in lung cancer. Sci Transl Med 2013;5:216ra177.

19. Yasuda H, Kobayashi S, Costa DB. EGFR exon 20 insertion mutations in non-small-cell lung cancer: preclinical data and clinical implications. Lancet Oncol 2012;13:e23-31.

20. Hirano T, Yasuda $\mathrm{H}$, Tani $\mathrm{T}$, et al. In vitro modeling to determine mutation specificity of EGFR tyrosine kinase inhibitors against clinically relevant EGFR mutants in non-small-cell lung cancer. Oncotarget 2015;6:38789-803.

21. Scagliotti G, Brodowicz T, Shepherd FA, et al. Treatmentby-histology interaction analyses in three phase III trials show superiority of pemetrexed in nonsquamous non-small cell lung cancer. J Thorac Oncol 2011;6:64-70.

22. Cohen M H, Cortazar P, Justice R, et al. Approval summary: pemetrexed maintenance therapy of advanced/ metastatic nonsquamous, non-small cell lung cancer (NSCLC). Oncologist 2010;15:1352-8.

23. Gergis C, Rangachari D, Fujii M, et al. EGFR-A763_ Y764insFQEA: a unique exon 20 insertion mutation that displays sensitivity to all classes of approved lung cancer EGFR tyrosine kinase inhibitors: J Clin Oncol 2019;15:e20593.

24. Lin YT, Liu YN, Wu SG, et al. Epidermal growth factor receptor tyrosine kinase inhibitor-sensitive exon 19 insertion and exon 20 insertion in patients with advanced non-small-cell lung cancer. Clin Lung Cancer 2017;18:324-32.e1.

Cite this article as: $\mathrm{Xu} C W$, Wang WX, Wang D, Wang QM, Pu XX, Zhu YC, Huang JH, Yu ZY, Cui ZL, Chen XH, Li JL, Fang Y, Wang H, Zhuang W, Lan SJ, Cai X, Zhang YB, Gao WB, Wang LP, She KL, Rao CZ, Zhou YF, Fang MY, Miao LY, Lei L, Lv TF, Song Y; on behalf of AME Lung Cancer Collaborative Group. Pemetrexed-based chemotherapy for non-small-cell lung cancer patients with EGFR exon 20 insertion mutation: a multicenter study. Transl Lung Cancer Res 2020;9(5):1853-1861. doi: 10.21037/tlcr-20-382 